\title{
LA IDENTIFICACION ANATOMICA DE MADERAS EN LATINOAMERICA ${ }^{1}$
}

\author{
Alirio Pérez Mogollón 2
}

\begin{abstract}
RESUMEN - A partir de la informazión bibliográfica incorporada a una base de datos, se esbozan algunas apreciaciones sobre la identificación de maderas en Latinoamérica. Se mencionanm los aspectos más resaltantes de esta materia en Venezuéla. Finalmente se indican algunas limitaciones, perspectivas y recomendaciónes de interés principalmente para los investigadores de Latinoamérica.
\end{abstract}

Palabras-clave: Anatomia de la maderas, Botánica en Latinoamerica.

\begin{abstract}
RESUMO - A partir da informação bibliográfica incorporada a uma base de dados, são esboçadas algumas considerações sobre a identificação de madeiras na América Latina. São mencionados os aspectos mais relevantes deste assunto na Venezuela. Finalmente são demonstradas algumas limitações, perspectivas e recomendações de interesse principalmente para os pesquisadores da América Latina.
\end{abstract}

Palavras-chave: Anatomia de madeiras, Botânica na América Latina.

\section{Introduccion}

Un análisis de la bibliografia sobre identificación de maderas de Latinoamérica significa una labor útil, amplia e interesante, especialmente para los investigadores de este campo. Actualizar los conocimientos y adicionar a las revisiones bibliográficas el creciente número de trabajos publicados en los último anõs, permite estimar el avance cualitativo y cuantitativo en esta materia. Sin pretensiones de ser exhaustivo en el tema, se presenta la información básica obtenida de una revision bibliográfica de los principales trabajos sobre anatomia e identificación de maderas realizados en Latinoamérica y disponibles o conocidos en Venezuela. Se incluyen también algunas publicaciones realizadas pero no disponibles en el momento de la revision por razones de distribucion restringida u otras causas. El propósito esencial es ofrecer una visión principalmente sobre el material bibliográfico en esta materia, conocido en Venezuela y no una revisión de todas las publicaciones existentes. En consecuencia, algunas inferencias esbozadas aqui, deben ser tomadas con reservas y deberian ser interpretadas como tendências predominantes en cuanto a productividade bibliográfica. Asi, mediante este inventario se logra una idea aproximada de la cuantia de la investigación realizada en el área, las formas como se presenta la información y la magnitud de la tarea aún por realizar.

\section{Material y Metodos}

Las referencias bibliográficas fueron incorporadas a una base de datos. Para la organización de la información se utilizó el dBASE III bajo el sistema operativo MS-DOS (Disk Operating System) en un computador KAYPRO equipado con un disco duro de 20 megabytes. Actualmente la base de datos tiene cerca de 900 registros, los cuales ocupan cerca de un megabyte. Cada registro contiene 18 campos para un total de 936 caracteres. Detalles sobre la estructura de la base de datos, el menú principal y el menú de consultas se indican respectivamente en las tablas 1-3. Como se puede inferir, se trata

\footnotetext{
${ }^{1}$ Trabalho apresentado no Workshop Internacional sobre Anatomia de Madeira dos Países Membros do Tratado de Cooperação Amazônica, realizado durante o XXXIX Congresso Nacional de Botânica, em Belém, PA, de 25 a $31 / 01 / 88$

2 Departament. Botánica Fac. Ciencias For. ULA. Mérida. Venezuela.
} 
de una base de datos en su etapa preliminar, en la cual cada referencia bibliográfica correponde a un trabajo publicado sin entrar en consideración sobre aspectos relativos al número de especies, gèneros y familias estudiados. Tampouco se discriminan dichos trabajos según la profundidad, extensión o esquemas descriptivos. En principio, se han incluido principalmente autores de latinoamérica. La base contiene también algunas referencias presentes en las revisiones bibliográficas realizadas por Araujo (1968), Gregory (1980) y la información que sobre esta materia ha sido divulgada en "Rodriguesia" y otras publicaciones análogas. Próximamente serán incorporadas algunas de las referencias contenidas en la revisión hecha por Tomazello (1987). A partir de la información recabada se esbozan algunas apreciaciones de caráter general.

Tabla 1 - Estructura de la base de datos

\begin{tabular}{rcr}
\hline Campo & Nombre del campo* & anchura \\
\hline 1 & NIFLA & 5 \\
2 & LUGAR & 12 \\
3 & TAXA & 18 \\
4 & TAXA2 & 18 \\
5 & CLAVE & 18 \\
6 & AUTOR1 & 20 \\
7 & AUTOR2 & 20 \\
8 & AUTOR3 & 20 \\
9 & AÃO & 5 \\
10 & TITULO & 180 \\
11 & IDIOMA & 5 \\
12 & EDITOR & 85 \\
13 & NUMPAG & 8 \\
14 & NUMREF & 7 \\
15 & NUMCUA & 7 \\
16 & NUMFIG & 7 \\
17 & RESUMEN1 & 250 \\
18 & RESUMEN2 & 250 \\
\hline Total & & 936 \\
\hline
\end{tabular}

* En secuencia vertical: No. IFLA, lugar, orden o familia género o especie, palabra clave, $1 \stackrel{\circ}{\circ} 2^{\circ}, 3{ }^{\circ}$ apellido... No. página, No.referencias, No. cuadros y/o tablas, No. figuras, resumen.

Tabla 2 - Menú Principal

(S) Salir del menú

(C) Consultar registros o fichas

(A) Agregar registros

(E) Eliminar registros

(D) Salir del sistema operativo

(Opción y luego (ENTER) 
Tabla 3 - Menú de Consultas

\begin{tabular}{ll}
\hline (L) & Listar fichas por autor \\
(G) & Listar por lugar geográfico \\
(F) & Listar por un taxón \\
(C) & Listar por palabra clave \\
(A) & Listar por año de publicación \\
(E) & Buscar por autor específico \\
(T) & Buscar por un taxón \\
(R) & Editar un registro específico \\
(M) & Volver al menú principal \\
\hline
\end{tabular}

(Opción y luego (ENTER)

\section{Resultados}

\section{Revisión bibliográfica}

Algunas estimaciones o inferencias de interés resultan de la revisión. La Fig. 1 muestra los paises latinoamericanos con mayor número de publicaciones acumuladas en el lapso 1930-1987. La predominancia de publicaciones brasileñas es evidente. Seguidamente aparecen México, Argentina y Venezuela. Los restantes países exhiben una producción mucho menor. Los investigadores que han contribuido más significativamente se indican en la Fig. 2. Algunas insuficiencias de datos en las referencias de la base impiden expresar en porcentaje o cifras más aproximadas algunas apreciaciones producto de la revisión. Sin embargo, la lectura del contenido de los trabajos y la utilización de algunas palabras claves en la base de datos, permiten esbozar las seguientes generalizaciones: a) la mayoria de las descripciones contiene tanto la parte microscópica como la macroscópica; b) algunos trabajos, menos frecuentes, con información orientada o concentrada hacia explicaciones de propriedades físicas, mecánicas y usos contienen solamente las descripción macroscópica; c) muchos trabajos mencionam la correspondiente muestra botánica; sin embargo algunos indican unicamentee la muestra de xiloteca y otros no hacen ninguna alusión a la procedencia de la muestra; d) en algunas publicaciones, especialmente de México, la descripción anatómica del leño se complementa com información estatística sustancial y referencia al material de herbario; e) en muchas publicaciones donde se intenta presentar diferenciaciones anatómicas de maderas es común encontrar una clave dicotómica, junto a la descripción ordinariaa. Pocos trabajos incluyen clave de entrada múltiple y muy excepcionalmente se encuentran descripciones según la lista estándar de características para uso computacional sugerida por la IAWA; predominan los enfoques principalmente descriptivos o de identificación; también, en menor cuantia, se presentan publicaciones que intentan lograr explicaciones o interpretaciones de caráter taxonómico. Trabajos con otra finalidad son mucho menos frecuentes. Una revisión del número de publicacionés sobre anatomia de maderas, de los paises miembros del Tratado de Cooperación Cooperación Amazónica indica de nuevo la notoria predominancia de Brasil y evidencia a la vez la magnitud de la tarea por realizar.

\section{Identificación de maderas en Venezuela}

De acuerdo a recientes estimaciones, Venezuela tiene alrededor de 3312 especies ar- 


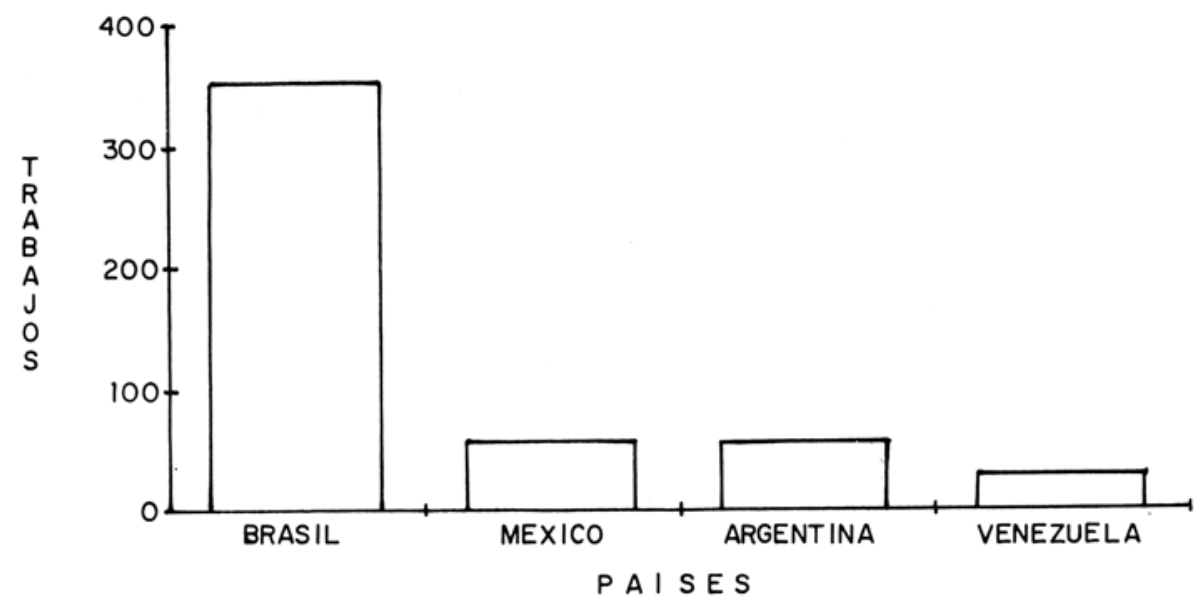

Figura 1 - Paises latinoamericanos con más de 20 publicaciones acumladas hasta 1987

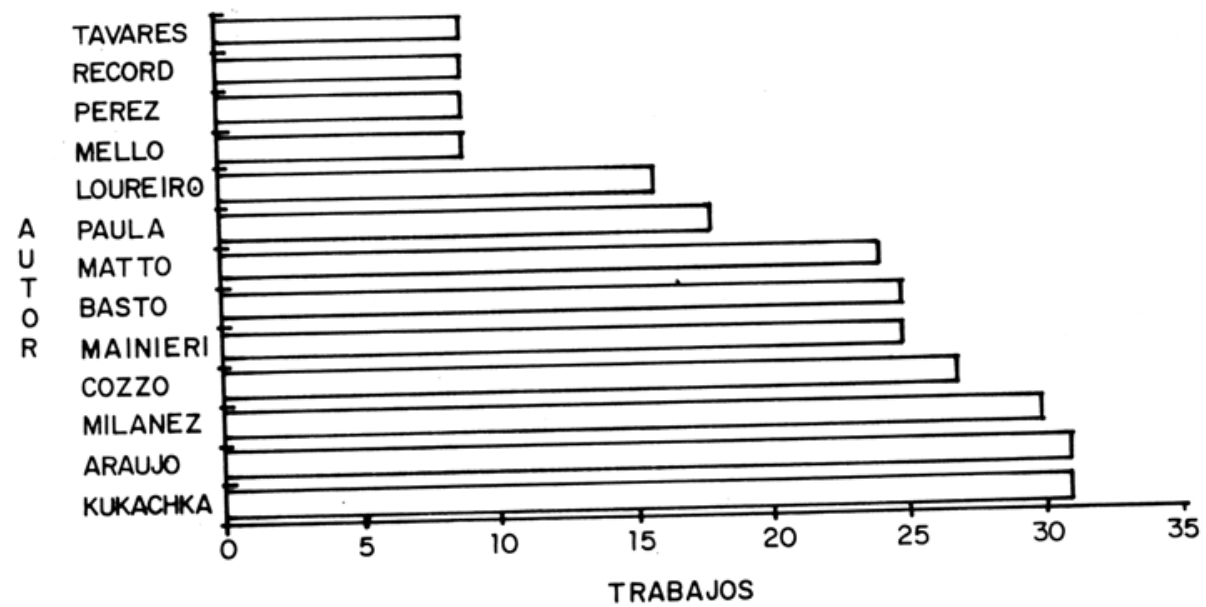

Figura 2 - Investigadores que han apartado significativamente. Lapso 1930-87 
bóreas nativas correspondientes a 696 géneros y 112 familas. Muchas de estas especies exhiben una distribución amplia abarcando la América Tropical en Sur, Centroamérica y las Antillas. Las labores de recolección, descripción e identificación de maderas se han orientado principalmente hacia las especies de las zonas de los LLanos Occidentales, Guayana y con menor intensidad, la Región Andina. La mayor colección de muestras leñosas se encuentra depositada en la Xiloteca MERw. Son aproximadamente 18000 especimenes correspondientes a 2364 especies (nativas y extranjeras), 908 géneros y 158 familias. Se dispone también de 25000 láminas o preparaciones microscópicas permanentes de tejidos leñosos. De las maderas recibidas por canje, resalta por su importancia, un conjunto de 500 muestras brasileñas de la colección de Krukoff.

Son numerosas las especies cuyas maderas no ha sido descritas ni aun colectadas. Algunos de los factores que más han influido en esto ha sido la inaccesibilidad de las zonas y la falta de recursos y apoyo logístico para realizar las exploraciones e incrementar las colecciones. La mayoria de la investigación anatómica descriptiva del leño ha sido realizada principalmente por Corothie (1948, 1966, 1967), Bascopé (1962), Mora (1974), Pérez (1969, 1974, 1987), Espinoza (1980, 1985a, 1985b) y Angarita (1981). Además de las descripciones macroscópica y microscópica, generalmente se presenta una clave dicotómica. Por otra parte, descripciones macroscópicas y clave de tarjetas perforadas para maderas de la Guayana venezolana ha sido elaboradas tomando como base la lista de características y el tipo de tarjeta puestos en práctica inicialmente por Clarke (1938) y perfeccionado luego en el Laboratório de Investigaciones de Productos Forestales en Londres. Siguiendo este esquema Pérez $(1977,1981)$ describe macroscópicamente el leño de aproximadament 136 especies de la Guayana venezolana y 148 especies de latinoamérica. Se presenta también una clave de tarjetas perforadas para la identificación macroscópica de maderas a nivel de família ((Pérez 1981). Con este propósito, se siguió el modelo de tarjetas empleado previamente por los botánicos Simpson y Janos (1974). Más recientemente, en Venezuela se han venido realizando descripcions anatómicas de maderas dicotiledóneas según la lista estándar de características adecuadas para la identificación asistida por el computador (Miller \& Baas 1981). Esta lista ha sido sugerida por la Asociación Internacional de Anatomistas de maderas (IAWA).

\section{Discusion y conclusion}

\section{Algunas limitaciones}

En general, se está consciente de la limitación natural propia de la estructura leñosa la cual muchas veces no ofrece una variabilidad suficiente para lograr una identificación precisa, particularmente en las categorias inferiores (especies y aun género). Por otra parte, son conocidas las limitaciones mayores o menores que ofrecen los diversos tipos de claves elaborados y usados en la identificación de maderas. Las descripciones mismas en su conjunto no siempre siguen un patrón descriptivo común que facilite la comparación e intercambio apropiado de información. En general, la comunicación entre los investigadores de la región, los intercambios de muestras leñosas y la distribución y difusión de los resultados de las investigaciones son deficientes. Algunos trabajos no hacen referencia al correspondiente material de herbario ni aportan datos que garanticen la autenticidad de la muestra descrita.

Obviamente junto a estas limitaciones o fallas, existe una serie de aspectos positivos y progresos evidentes en los estudios anatómicos de maderas. Básicamente, el señalamiento concreto de estas limitaciones tiene el único propósito de estimular su análisis y recabar apreciaciones sobre las posibles orientaciones y correctivos que pudieran ser incorporados en las investigaciones futuras. 


\section{Perspectivas y recomendaciones}

Los resultados de las discusiones sobre esta materia deberán ofrecer vias y acciones que contribuyan al progreso de los estudios tendentes a lograr un mejor conocimiento anatómico de nuestras maderas y una mayor cooperación entre los países o instituciones involucrados en esta misión.

El conocimiento de nuestras maderas es un reto de interés común pra Latinoamérica. En general, se dispone de una riqueza florística abundante, variada, heterogénea; muchas especies tienen una distribución natural amplia; las facilidades de comunicación entre los países es cada vez mayor; las iniciativas hacia una creciente coparticipación en proyectos de interés común se han venido incrementando gradualmente. En este contexto, se presentan los proyectos Andinos de Desarrollo Tecnológico en el área de los Recursos Forestales Tropicales (PADT-REFORT) de la Junta del Acuerdo de Cartagena, el Tratado de Cooperación Amazónica con sus eventos importantes que realiza e instituciones meritorias. En este sentido, aun con las limitaciones económicas y financieras actuales, las perspectivas para realizar empresas comunes son prometedoras.

Parece conveniente efectuar acciones tendentes a consolidar un sistema de información que tome en cuenta los siguientes aspectos:

\section{Respecto a las descripciones anatómicas de maderas}

1.1. Parece deseable unificar los esquemas descriptivos de la estructura leñosa, irrespectivamente de los propósitos de cada investigación (tecnologia, taxonomia, etc). Con frecuencia se observa, dependiendo del enfoque o propósito de la investigación, variaciones en las formas descriptivas. Un buen punto de partida tendente a la unificación pareciera ser la lista estándar de la IAWA. En Venezuela las descripciones más recientes siguen dicha lista.

1.2. Es importante acompañar cada descripción del leño, con los correspondientes datos referentes a muestras de herbario, sinonimia, distribución, etc.

\section{Referente a la coparticipación e intercambio de información}

2.1. Incrementar las acciones orientadas a lograr una coparticipación apropiada en investigaciones de interés común. Ejemplo, descripción anatómica de la madera de especies arbóreas discriminadas por categorias taxonómicas, grupos de maderas con usos o propiedades similares e por regiones geográficas.

2.2. Asegurar una mayor fluidez en el intercambio de información bibliográfica y de muestras de maderas y de herbario. Publicaciones como el boletin de la IAWA $\mathrm{Y}$ otras de latinoamérica pueden ayudar mucho en relación a estos aspecto.

2.3 Intensificar la comunicación y cooperación apropiadas entre los investigadores, en función de aglutinar esfuerzos ante situaciones de responder consultas sobre la identidad de muestras leñosas.

3. Finalmente, parece conveniente analizar la posibilidad de crear o fortalecer un centro de documentación con un sistema de información apropriado, con capacidad para atender labores de divulgación y consultas de benefício colectivo. Un centro que pueda recibir y reseñar las publicaciones que sobre la materia se produzcan, y a la vez organizar, procesar y divulgar dicha información.

\section{Agradecimientos}

Al Ing. O. Encinas por su valiosa ayuda en el trabajo computacional de creación y operatividad de la base de datos. 


\section{Referências Bibliográficas}

ANGARITA, T. N. 1981. Estudio anatómico de algunas especies de la familia Bombacacear. Mérida, Universidad de los Andes, Fac. de Ciências Forestales, 46p.

ARAÚJO, P.A. M. 1968. Bibliografia sobre anatomia de maderas. Anu. Bras. Econ. Flor. 19(9):243-332.

BASCOPE, F. 1962. Clave de identificación macroscópica de algunas maderas dicotiledóneas de Latinoamérica. Mérida. Inst. For. Latinoamer. Invest. 9:16-103.

CLARKE, S. H.A. 1938 multiple-entry perforated card key with special reference to the identification of hardwoods. New Phytol. 37(4):369-374.

COROTHIE, H. 1948. Maderas de Venezuela. Caracas. Inst. Bot. MAC. 320 p.

COROTHIE. H. 1966. Estructura anatómica de 30 maderas de la Guayana venezolana y clave para su identificación. Mérida, Universidad de los Andes, Fac. de Ciencias Forestales, 93p.

COROTHIE. 1967. Estructura anatómica de 47 maderas de la Guayana venezolana y clave para su identificación. Mérida, Universidad de los Andes, Fac. de Ciencias Forestales, $125 \mathrm{p}$.

ESPINOZA, P. A. 1980. Estudio anatómico de la madera Cedrela. Mérida, Universidad de los Andes, Facultad de Ciencias Forestales, 125p.

ESPINOZA P.A. \& A. QUINTERO, A. 1985a. Codificación y creación de archivos de características anatómicas en maderas dicotiledóneas para uso computacional. Mérida, Universidad de los Andes, Fac. de Ciencias Forestales, 45p.

SPINOZA P.A. 1985b. Identificación de maderas dicotiledóneas asistida por microcomputador. Mérida, Universidad de los Andes, Facultad de Ciencias Forestales, 92p.

GREGORY, M. 1980. Wood identificacion: an annotated bibliography. IAWA Bull. n. S. $1: 3-41$.

MILLER, R.B. et al. 1981. Standar list of characters suitable for computerized hardwood identificacion: IAWA Bull n.s. 2: 2-3,99-129.

MORA, J.J. 1987. Características tecnolőgicas de 37 maderas venezolanas. Mérida, Universidad de los Andes, Fac. de Ciencias Forestales, 151 p.

PEREZ M.A. 1974. Identificación macroscópica de algunas maderas de los Llanos Occidentales de Venezuela. Mérida, Universidad de los Andes, Fac. de Ciencias Forestales, 39p.

PEREZ M.A. 1973. Estructura anatómica de 37 maderas de la Guyana venezolana y clave para su identificación. Acta. Bot. Venez. 8(1-4):9-110.

PEREZ M.A. 1977. Identificación macroscópica de algunas maderas de la Guayana venezolona y clave para su identificación. Mérida, Universidad de los Andes, Facultad de Ciencias Forestales, 56p.

PEREZ M.A. 1981. Identificación macroscópica de algunas maderas de Latinoamérica. Clave para la identificación macroscópica de maderas a nivel de familia. Mérida, Universidad de los Andes, Fac. de Ciencias Forestalés, 83p.

PEREZ M.A. 1988. Bibliografia sobre anatomia de maderas latinoamericanas. Mérida, Inst. For. Latinoamer. v.1

SIMPSON, R. \& JANOS, D. 1974. Punch card key to the families of dicotyledons of the western hemisphere south of the United States. Chicago, Illinois, Field Mus. Nat. Hist. 16p.

TOMAZELLO, F.M. 1987. Bibliografia sobre anatomia e identificação de madeiras. Piracicaba, Universidade de São Paulo, 47p. 